\title{
Primary step for endoscopic sinonasal tract and anterior skull base robotics
}

\author{
Vincent Trévillot ${ }^{1,2}$, Etienne Dombre ${ }^{2}$, Philippe Poignet ${ }^{2}$, Rafaël Sobral ${ }^{2}$, Olivier Tempier ${ }^{2}$, \\ Benoît Herman $^{3}$, Renaud Garrel ${ }^{1}$, Louis Crampette ${ }^{1}$ \\ ${ }^{1} \mathrm{CHU}$ de Montpellier, France \\ ${ }^{2}$ LIRMM-UMR 5506 CNRS-UM2, France \\ ${ }^{3}$ CEREM, Université catholique de Louvain - F.R.S.-FNRS, Louvain-la-Neuve, Belgium \\ E-mail address: v.trevillot@gmail.com, dombre@lirmm.fr, poignet@lirmm.fr, tempier@lirmm.fr, \\ benoit.herman@uclouvain.be,Sobral@lirmm.fr, r-garrel@chu-montpellier.fr, 1-crampette@chu-montpellier.fr
}

\begin{abstract}
Introduction: Surgeons have evolved a lot their surgical procedures in sinus surgery and are now able to resect malignant tumors. These progresses are now leading new difficulties like impairing vision (bleeding and LCR flow) and necessity of multiple simultaneous tasks. With the aim of designing a new endoscope-holder, primary step was to characterize the surgeon gesture, the kinematics, and the type of man-machine interface acceptable by the surgeon.

Methods: We worked on thirteen sinonasal tracts of cadaver heads. Surgical procedures were: opening all the sinuses, the carotid, the sella turcica, the lamina papyracea and the anterior skull base. We used conventional instruments which were instrumented with a force-torque sensor and a navigation system. Then we have experimentally evaluated robots with three different kinematics and two types of man-machine interfaces.

Results: We collected enough position and force data as well as kinematics constraints and interface requirements to specify a robot for endoscopic sinus surgery.
\end{abstract}

\section{Introduction}

Before 1970, open surgery was the only way for surgical approach to the sinonasal tract. This kind of surgery was reserved for organic disease because of its difficulty, invasiveness and painfulness. In the 70's, Messerklinger and Stammberger were the first surgeons to use an endoscope so as to perform minimally invasive approach to the sinuses and to treat functional disease [1]. Nowadays, surgeons are still using an endoscopic approach, and technology has evolved allowing treatment of benign and malignant tumors. Surgeons are able to open all the sinuses (maxillary sinus, ethmoid sinus, frontal sinus and sphenoid sinus), and to have access to the brain through the sino-nasal tract and the anterior and middle-stage of the skull-base. To facilitate the procedure, new equipments have been introduced in the operating room (OR) like video-camera system and navigation system (figure 1). Practically, the patient is lying down on the back, the surgeon is standing on his/her right side and holds a rigid endoscope in his/her left hand (diameter: $4 \mathrm{~mm}$, length: $17 \mathrm{~mm}$, orientation: $0^{\circ}, 30^{\circ}$ or $70^{\circ}$ ), while the right one is holding a suction instrument that is used for breaking the different structures and sucking blood and secretions. In case of important bleeding or cerebrospinal flow, the surgeon keeps an endoscope with his/her left hand but has to frequently interchange the suction instrument and the grasper in his/her right hand. In order to overcome this difficulty, surgeons would need three hands: one for holding the endoscope, one for holding a suction instrument and the third for holding a grasper instrument. A natural solution is obviously to involve two surgeons, typically an ENT surgeon together with a neurosurgeon who may bring complementary knowhow. This solution has been improved by Briner et al. [2] who analyzed and compared the four-hand and two-hand surgeries, and proved that the former saves $21 \%$ of the operation time for ethmoidectomy.

This is an Open Access article distributed under the terms of the Creative Commons Attribution-Noncommercial License 3.0, which permits unrestricted use, distribution, and reproduction in any noncommercial medium, provided the original work is properly cited. 
Castelnuovo et al. described in [1] their experience and results. According to them, the four-hand surgery is the only one that may allow large tumor resections. Unfortunately, it is very expensive, time-consuming and needs a great complicity and a great experience of the two surgeons. Another way could be the use of a robotic endoscope-holder. This robotic solution is very attractive. However, the literature review showed us first that the da Vinci robot (Intuitive Surgical, USA) was very invasive and cumbersome [3-6] for skull base surgery, then that other exploratory prototypes were very cumbersome around the tip of the nose, giving only limited access to the sinonasal tract and the skull-base [7-12]. This has motivated our work to design a compact, ergonomic and safe robotic endoscope-holder providing a stable image and dedicated to the sinonasal tract, and the anterior and middle-stage skull-base. This paper describes the three steps of the design: motion analysis for specifying the robot, design of a kinematics satisfying the motion requirements, and analysis of the man-machine interface.

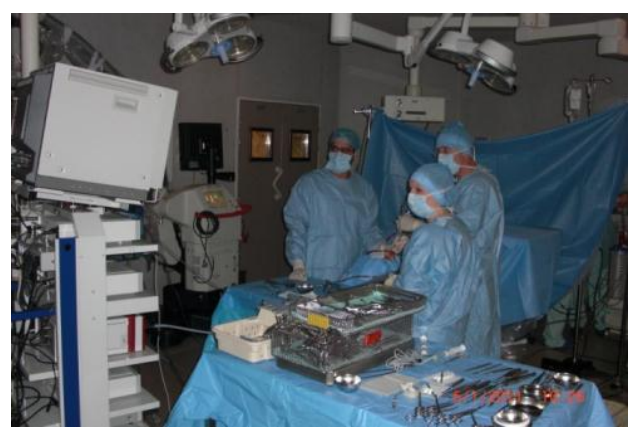

Figure 1 : Operating room for skull-base surgery

\section{Specifications}

The first step of our work was to record data about the surgeon gesture. Therefore, we have quantified accurately the parameters involved in the design of the robot to comply with two constraints: i) the robot should not limit the surgeon's motion; ii) it should be safe for the surgeon and the patient. We made experiments to record basic data describing the position and orientation of the tools during ENT surgery as well as the maximal forces exerted by the surgeon on the instruments. An ENT resident operated 13 cadaver sinonasal tracts fixed by freezing. Prior to surgery, high resolution computed tomography (CT) has been performed on the heads (slice thickness of
$0.625 \mathrm{~mm}$ for native images and $1 \mathrm{~mm}$ for reconstruction in the sagittal, axial and frontal planes). In order to accurately register pre-operative and peroperative data, we designed a head-holding system. Then, each head was mounted with 5 PVC fiducial markers screwed in the anterior part of the skull. We used PVC markers because PVC does not generate artifact and is well visualized on bone window CT images. The registration procedure consisted in a robust point to point step refined by a surface morphing step. An Easytrack navigation system (Atracsys, Switzerland) based on two infra-red cameras was used together with specific markers stuck on standard instruments: a $30^{\circ}$ endoscope (diameter: 5 $\mathrm{mm}$, length: $17 \mathrm{~mm}$, Wolf, USA), and a suction device (Medtronic, USA). We also instrumented these instruments with force-torque sensors (respectively Nano 43 and Nano 45, Schunk GmbH, Germany). With such a setup, we were able to localize the tip of both instruments into the CT-scan images, to record their position and orientation, and to measure forces applied on them during the surgery. A dedicated visual display has been developed (figure 2) returning to the surgeon the navigation data (current position of the tip of the suction instrument in the CT data projected in the sagittal, axial and frontal planes), and the module of forces delivered by the force-torque sensors: the endoscope sensor gives data about normal friction applied on the endoscope, and the suction instrument sensor gives data about minimally forces to open functional structures and to damage vital ones. We also display the endoscopic data as well as a video of the gesture of the surgeon's hand and the motion of the instrument. This is done for analysis of instrument interactions during surgery. All the data, as well as the angular velocities in the three planes and the penetration of the endoscope in the nostril, are recorded for future processing.

The experimental approach was basically the same as conventional surgery: the ENT resident, standing on the right side of the patient, is holding the endoscope with his left hand, and is using the suction and the grasper instrument with his right hand. He made ethmoidectomy surgery that requires opening whole sinuses (functional structures). After that, he broke the internal carotid, the sella turcica, the lamina papyracea, and the anterior skull-base (vital structures) to test their breaking resistance. Data mining was made 
at the Laboratory. It consisted in extracting relevant data from videos, force-torque sensors and navigation system, ensuring their consistency and searching the reasons of non-consistency when happen. It turned out that the future robotic endoscope-holder for sinus and skull-base endoscopic surgery should satisfy the following constraints: friction forces up to $20 \mathrm{~N}$; maximal forces to prevent structures $40 \mathrm{~N}$; orientation angles up to $66^{\circ}$ in the sagittal plane and $68^{\circ}$ in the axial plane; rotation about the endoscope axis up to $71^{\circ}$; angular velocities up to $43 \% \mathrm{~s}$ in the sagittal plane, $56 \%$ in the axial plane and $57 \%$ s about the endoscope axis; penetration in the nose up to $100 \mathrm{~mm}$. We also tried to determine whether the nostril could be considered as a fixed point for the endoscope. This could imply to design a dedicated kinematics with a socalled "Remote Center of Motion" (RCM) about which the endoscope would be constrained to rotate. We found for example that there is a pseudo-fixed point when opening the maxillary sinus, another one when opening the frontal sinus. For each sinonasal tract, the pseudo-fixed point moves in a small fixed box, the average edge of which being about $2 \mathrm{~mm}$.

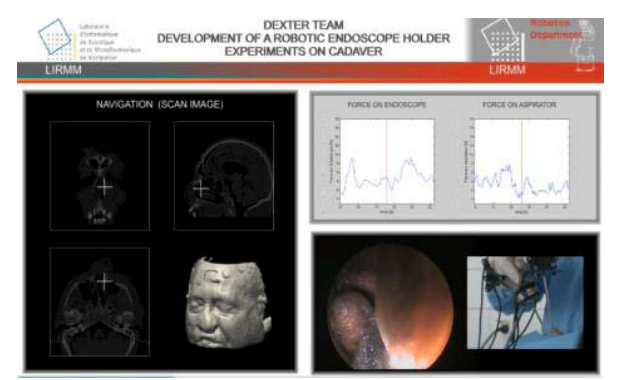

Figure 2: Visual display of navigation data, forces, endoscopic and video data

\section{Kinematics}

First, a literature review of kinematics used in the OR for sinonasal tract surgery showed that several prototypes have been developed [13-19] but none of them fully satisfies requirements in terms of compacity and ergonomy. Thus we have evaluated three types of kinematics on cadaver heads. The first one is EVOLAP (figure 3), a prototype built for minimally-invasive laparoscopic surgery [20] at University of Louvain la Neuve. It has two active Degrees Of Freedom (DOF) and generates a RCM. The main manipulator has a particular kinematic structure composed of three orthogonal parallelograms that translates the end- effector on the surface of a half-sphere. A local manipulator composed of a gimbal with two passive DOF is attached at the distal end of an adjustable passive arm attached to the main manipulator. The translation of the laparoscope to produce the in-out motion ("zoom" of the video images) and the rotation about its axis are not controlled in the current version of the robot. We designed a new local device to reduce its size as required. The second robot is a six active DOF industrial robot (VIPER from Adept Technology, USA) (figure 4). The endoscope is fixed to the robot through a $40 \mathrm{~cm}$ curved link devoted to free the nostril region. The third kinematics is a mix of the EVOLAP and the VIPER kinematics. The VIPER holds the local manipulator of EVOLAP. We modified the control of the VIPER to constrain the endoscope motion on the surface of a half-sphere. Doing that the penetration of the endoscope (the zoom) is actively controlled, which, as above mentionned, is not the case with the current version of EVOLAP.

One limitation of the EVOLAP kinematics is that it is difficult to achieve a proper control of the endoscope when it is not within the nose. The HYBRID kinematics offers the advantage of controlling the penetration of the endoscope. The VIPER kinematics allows to control both penetration and orientation of the endoscope. However from the safety point of view, alignment errors with this solution could severely damage anatomical structures, which is not the case with the former ones as the gimbal frees the orientation of the endoscope.

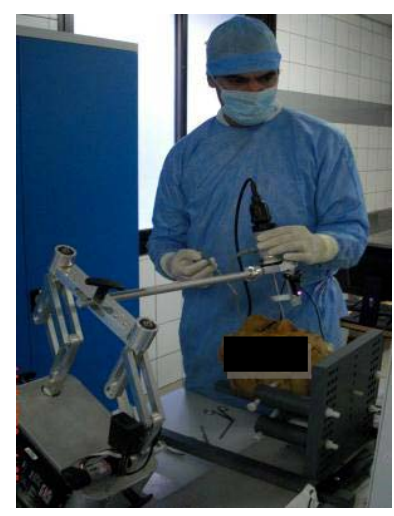

Figure 3 : EVOLAP kinematics 


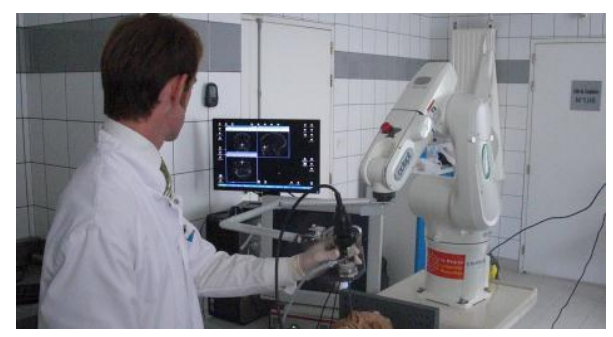

Figure 4 : Hybrid kinematics

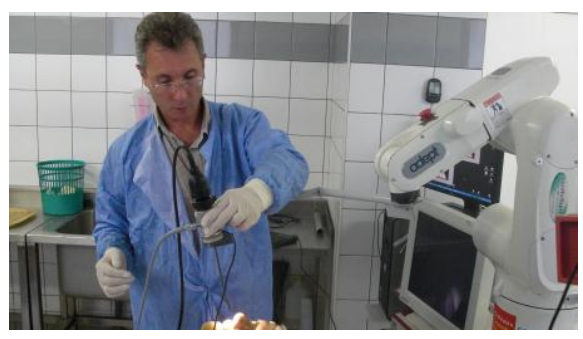

Figure 5: VIPER kinematics

\section{Man-machine interface (MMI)}

Several types of interfaces can be used to control endoscope-holders: voice, head motion, foot pedal, or joystick as highlighted in [20-22]. These interfaces come to control the endoscope-holder in a so-called "telemanipulation" mode: the surgeon acts on a device that in turn acts on the robot. Another way is to implement an automatic control of the robot through visual servoing [23]: markers are stuck on the distal part of the instruments and are tracked by the endoscope such that they remain in the center of the image. It is however difficult to assess the safety of such a method as markers may be temporarily hidden by blood and smoke or by another instrument. An interesting alternative is "comanipulation". In a comanipulation mode, the surgeon moves manually the endoscope as in conventional surgery. The difference is that the robot follows the motion of the endoscope and maintains it in position when the surgeon does not hold it anymore. This requires a force-torque sensor and an appropriate force control scheme. The difficulty is to obtain a true zero-force servoing providing enough "transparency" such that the surgeon does not "feel" the weight and inertia of the robot. Two modes have been tested:

- Telemanipulation: a simple PlayStation joystick was used which allowed to control omnidirectional displacements of the endoscope and real time velocity adjustment.

- Comanipulation: as above mentioned, a forcetorque sensor is used which is mounted between the endoscope and a dedicated handle.

It turned out that the best MMI for sinonasal endoscopic robotic surgery is the comanipulation mode. The reason is that it is the most natural way for the surgeon to pull in and out the endoscope from the nostril, gesture which is done rather frequently (we counted an average of 50 for the ethmoidectomy). Moreover, it allows them to let the endoscope in a rigid position to free one hand when necessary.

\section{Conclusion}

This work allowed us to specify the future endoscope holder dedicated to sinonasal tract and anterior and middle stage skull base surgery. From the experimental studies,sinonasal we are now in the process of choosing a kinematics - a standard 6-DOF robot with a dedicated local semi-active manipulator - and a MMI based on comanipulation.

\section{Bibliography}

[1] P. Castelnuovo, I. Dallan, P. Battaglia, et M. Bignami, «Endoscopic endonasal skull base surgery: past, present and future », European Archives of OtoRhino-Laryngology: Official Journal of the European Federation of Oto-Rhino-Laryngological Societies (EUFOS): Affiliated with the German Society for OtoRhino-Laryngology - Head and Neck Surgery, vol. 267, nº. 5, p. 649-663, mai 2010.

[2] H. R. Briner, D. Simmen, et N. Jones, « Endoscopic sinus surgery: advantages of the bimanual technique ", American Journal of Rhinology, vol. 19, n $^{\circ}$. 3, p. 269-273, juin 2005.

[3] E. Y. Hanna, C. Holsinger, F. DeMonte, et M. Kupferman, « Robotic endoscopic surgery of the skull base: a novel surgical approach », Archives of Otolaryngology-Head \& Neck Surgery, vol. 133, $\mathrm{n}^{\circ}$. 12, p. 1209-1214, déc. 2007.

[4] J. Y. K. Lee et al., " Transoral robotic surgery of the skull base: a cadaver and feasibility study ", ORL; Journal for Oto-Rhino-Laryngology and Its Related Specialties, vol. 72, no . 4, p. 181-187, 2010.

[5] R. R. McCool, F. M. Warren, R. H. Wiggins, et J. P. Hunt, "Robotic surgery of the infratemporal fossa utilizing novel suprahyoid port ", The Laryngoscope, vol. 120, $\mathrm{n}^{\circ} .9$, p. 1738-1743, sept. 2010.

[6] B. W. O'Malley et G. S. Weinstein, «Robotic anterior and midline skull base surgery: preclinical investigations », International Journal of Radiation 
Oncology, Biology, Physics, vol. 69, nº. 2, p. S125128, 2007.

[7] K. W. G. Eichhorn et F. Bootz, « Clinical requirements and possible applications of robot assisted endoscopy in skull base and sinus surgery ", Acta Neurochirurgica. Supplement, vol. 109, p. 237240, 2011.

[8] C. Nimsky, J. Rachinger, H. Iro, et R. Fahlbusch, «Adaptation of a hexapod-based robotic system for extended endoscope-assisted transsphenoidal skull base surgery ", Minimally Invasive Neurosurgery: MIN, vol. 47, $\mathrm{n}^{\circ}$. 1, p. 41-46, févr. 2004.

[9] C.-A. O. Nathan, V. Chakradeo, K. Malhotra, H. D'Agostino, et R. Patwardhan, « The voice-controlled robotic assist scope holder AESOP for the endoscopic approach to the sella », Skull Base: Official Journal of North American Skull Base Society ... [et Al, vol. 16, $\mathrm{n}^{\mathrm{o}}$. 3, p. 123-131, août 2006.

[10] G. Strauss et al., " [Manipulator assisted endoscope guidance in functional endoscopic sinus surgery: proof of concept] », Hno, vol. 55, n .3 , p. 177-184, mars 2007.

[11] J. Wurm et al., "[Development of an active robot system for multi-modal paranasal sinus surgery] », Hno, vol. 53, no. 5, p. 446-454, mai 2005.

[12] T. Xia et al., " An integrated system for planning, navigation and robotic assistance for skull base surgery », The International Journal of Medical Robotics + Computer Assisted Surgery: MRCAS, vol. $4, n^{\circ} .4$, p. 321-330, déc. 2008

[13] S. Aiono, J. M. Gilbert, B. Soin, P. A. Finlay, et A. Gordan, «Controlled trial of the introduction of a robotic camera assistant (EndoAssist) for laparoscopic cholecystectomy », Surgical Endoscopy, vol. 16, n . 9, p. 1267-1270, sept. 2002.

[14] A. Arora et al., "Clinical applications of Telerobotic ENT-Head and Neck surgery », International Journal of Surgery (London, England), vol. 9, n ${ }^{\circ} .4$, p. 277284,2011
[15] K. Bumm et al., " An automated robotic approach with redundant navigation for minimal invasive extended transsphenoidal skull base surgery ", Minimally Invasive Neurosurgery: MIN, vol. $48, \mathrm{n}^{\circ} .3$, p. 159-164, juin 2005 .

[16] P. A. Federspil, B. Plinkert, et P. K. Plinkert, «Experimental robotic milling in skull-base surgery », Computer Aided Surgery: Official Journal of the International Society for Computer Aided Surgery, vol. 8, $\mathrm{n}^{\circ} .1$, p. 42-48, 2003.

[17] T. Haidegger et Z. Benyó, "[Surgical robotics in neurosurgery] ", Orvosi Hetilap, vol. 150, n .36 , p. 1701-1711, sept. 2009.

[18] J. E. Jaspers, K. T. Den Boer, W. Sjoerdsma, M. Bruijn, et C. A. Grimbergen, «Design and feasibility of PASSIST, a passive instrument positioner », Journal of Laparoendoscopic \& Advanced Surgical Techniques. Part A, vol. 10, nº. 6, p. 331-335, déc. 2000.

[19] A. C. Lehman et al., « Natural Orifice Translumenal Endoscopic Surgery with a miniature in vivo surgical robot », Surgical Endoscopy, vol. 23, nº. 7, p. 16491649, avr. 2009.

[20] B.Herman, B. Dehez, K. Tran Duy, B. Raucent, E. Dombre, S. Krut, «Design and preliminary in vivo validation of a robotic laparoscope holder for minimally invasive surgery », The Int. J. Medical Robotics and Computer Assisted Surgery, Vol 5, 2009, pp. 319-326.

[21] A. A. Gumbs, F. Crovari, C. Vidal, P. Henri, et B. Gayet, "Modified robotic lightweight endoscope (ViKY) validation in vivo in a porcine model ", Surgical Innovation, vol. 14, $\mathrm{n}^{\circ} .4$, p. 261-264, déc. 2007.

[22] L. K. Jacobs, V. Shayani, et J. M. Sackier, " Determination of the learning curve of the AESOP robot », Surgical Endoscopy, vol. 11, n ${ }^{\circ} .1$, p. 54-55, janv. 1997. 